\title{
In the Classroom
}

\section{Progress in Practice: The Synergy Derived From Knowing Pedagogy as Well as Chemistry*}

BRIAN P. COPPOLA

Department of Chemistry The University of Michigan Ann Arbor, MI 48109-1055, USA bcoppola@umich.edu

\section{Subject} matter mastery is the beginning, not the end, of effective instruction.

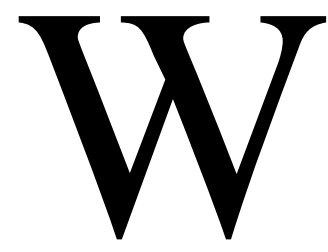

hat is the value that comes from consciously and explicitly linking what we know about chemistry with what we do in the classroom? It is tempting to dismiss this question because we are uncomfortable with the implication that there are times when what we do in the classroom is not informed by our personal understanding of chemistry. Yet instructors of

*Individuals involved in curriculum design often introduce new, modified, or applied ideas about instruction that span from classroom methods to philosophies of education. In this series, we examine progress in chemical education that is related to actual practices, and where many recommendations have originated from areas in higher education that exist alongside of and overlap with chemistry. Rather than an exhaustive review, we will select examples, background, and vocabulary that may either invite interested newcomers to explore a different area in their teaching, or provide language and precedent for individuals who wish to contextualize ideas they have developed independently.

-Brian P. Coppola, Series Editor 
introductory chemistry courses often lack the personal understanding, especially the kind that comes from laboratory experience, for significant parts of the course. An experienced general chemistry instructor, for example, probably understands the practical expectations of teaching this subject better than anyone who has recently graduated with a Ph.D. in physical or inorganic chemistry. Although the merits of this situation are worth reflecting on at another time, a reasonable operational assumption is that a substantial portion of the introductory program is defined by its own existence rather than as an identifiable area of specialization. The general chemistry curriculum is flexible to the degree that it can accommodate a variety of backgrounds in its instructors, yet it is constrained by the historical inertia that has defined it. To a lesser yet still significant extent, beginning instructors of organic chemistry face the same problem when their understanding of more specialized topics (such as the synthesis of heterocyclic compounds, transition metal organometallics, carbohydrate and peptide chemistry) is limited by their inexperience in those areas. Organic chemists might have only studied these topics as a part of their own introductory or intermediate instruction, and the textbook in use could be their primary source of information. Consequently, introductory chemistry instruction is filled with its own "urban myths", or perhaps they are parables [1] passed down from author to author, about chemical phenomena that may or may not stand up to the scrutiny of contemporary understanding. Sometimes this is by design; for instance, demonstrating some general features about macroscopic properties can be done by using simplifications like the ideal gas assumptions or with the use of concentration instead of activity. Intentional simplifications that use less sophisticated models to explain phenomena at an adequate level of complexity are commonplace (in fact, this is not a bad interpretation of Occam's Razor as it applies to science in general). This may be analogous to the way our colleagues in physics begin college instruction with Newtonian mechanics, or the way chemists can successfully use valence bond models for molecular structure to do a prodigious amount of chemistry without ever invoking a Hamiltonian operator. Problems can arise, however, whenever an instructor's depth of understanding of a subject is only marginally different than the simplified version of it. Agassi [2] offers a sobering view on the way some writers of introductory textbooks "mislead the innocent reader" (implying that unwary instructors will sometimes mislead learners). He laments that individuals who ultimately choose science do so in spite of their formal education and he refers to them as "those who survive the injury of the science textbook."

Sometimes what begins as a well-intended simplification ends up as an unnecessary complication. The first lesson in choosing between competitive mechanistic pathways that students encounter is the manifold of reactions represented by the uni- and 
bimolecular nucleophilic substitution and elimination pathways in organic chemistry $\left(S_{N} 2, E 2, S_{N} 1, E 1\right)$. Students tend to perceive these as exclusionary predictive choices; that is, the selection of one pathway, deemed to be determinable for any given set of reagents, will overwhelm the other possible pathways. Students feel compelled to "figure out" which pathway to use in much the same way they have been trained to select between mathematical formulas (Do I use this equation here or that one?). The underlying usefulness of the substitution-elimination manifold is that Nature does not provide the clear distinctions students crave, and that an understanding of competition between pathways is key to understanding actual experimental results. Here, the simplification that implies exclusivity rather than competition is counterproductive to an understanding of the fundamental relationships. In cases such as this one, there is an instructional challenge, the solution of which draws from both a deep understanding of the subject matter involved and an understanding of ways to involve an inexperienced learner with the more mature skills needed to understand a complicated topic at a complex level.

An underlying assumption in college instruction has been that the subject matter expertise of a faculty member is a necessary and sufficient prerequisite to good teaching, a notion that Agassi [2] would strongly support. Of course, practice and experience improve classroom skills, but the traditional definition of a teacher-scholar still hinges on the "scholar" component. This emphasis on an individual's expertise in the subject, while appropriate, is only the first half of the story of effective instruction. Colleges and universities are filled with subject matter experts who nonetheless struggle with their teaching duties, even after extended periods of time. Knowing how to design effective instruction in the subject that also achieves objectives for higher order learning (predicated on the belief that there is something in addition to chemistry to learn from a chemistry course) goes beyond only the mastery of the subject matter. It requires knowledge of how to blend understanding of the content with awareness of and experience with various teaching models, strategies, and materials. For instance, if we agree that becoming comfortable with the uncertainty of science is an overarching educational goal, then our deep knowledge of chemistry is crucial to the next step. The deeper and more elaborate our understanding is, the more it will allow us to pick and choose among the variety of situations we know of in order to create a situation that will make this understanding accessible to students. 
An illustrative example is appropriate at this point. One important lesson about solving structural chemistry problems is that chemists can sometimes only narrow their answer down to a selection between equally viable options. We have designed our first-year Honors laboratory program in a way that tests the scientific 'courage' of our students. They obtain empirical data that are consistent with two rational and unresolvable interpretations. Although the Friedel-Crafts acylation of 2-chlorotoluene gives a single major product, even a prediction about its identity based on standard textbook information is inconclusive. The 4-6 week project includes the opportunity to perform comparative experiments on other substrates. Students also do computational analyses such as constructing Frontier Molecular Orbitals and determining electrophilic susceptibilities for the six carbon atoms that comprise the aromatic ring. Throughout the activity, students experience an underlying conflict between maintaining the scientific integrity of two (or more) viable solutions and expecting a single correct answer (derived from their preconceptions about the nature of science). In designing this laboratory course, it was important to make a distinction between using any one of the hundreds of examples that are, to us, equally representative of a given concept and creating a situation that is designed to maximize student learning. The more we can understand and adopt our students' perspective on their understanding, the more we can be aware of all the factual information and experience we cannot fairly expect them to use (it is exactly our experience they lack!) when learning our subject.

Over the last ten years, the connection between one's understanding of the subject matter and the design of effective instruction has been articulated in a way that goes beyond content mastery, yet appropriately depends on it [3-5]. Effective instruction will naturally draw on the depth of understanding and expertise in the subject that an instructor brings to a course. Beyond this, it also includes insights on how learning the subject matter fosters a given critical skill, and which of many examples or strategies are best suited to develop such skills in a given student population. This additional ability of instructors is called pedagogical content knowledge (PCK). Originally formulated by Shulman in the areas of precollege science education and teacher preparation [6, 7], pedagogical content knowledge has been simply described as "a teacher's understanding of how to help students understand specific subject matter" [8]. An instructor's ability to differentiate the most compelling examples (to a trained expert) of the substitutionelimination manifold from the best ways to help inexperienced learners understand it is the unique domain of pedagogical content knowledge. 
The development of pedagogical content knowledge is not isolated from an instructor's beliefs about his or her role in the classroom. To some instructors posing the inevitable exceptions to some generalization in order to have students examine the fuzzy edges of a theory or its model is a way to try to ensure that the learners have examined ideas in a comprehensive way. For some students this will be a delightful puzzle filled with the reward of self-discovery. These students remind us of ourselves, at least as far as our subject matter is concerned. Not everyone is like us (or them), and our success in teaching our course will hinge on whether or not we can also accept the majority of our students, those who are not like us at all, and also provide for them. This perspective is not a compromise of the instructional destination, which is the development of an appropriate set of skills and an understanding of some factual subject matter and its origins. It does, however, require instructors to reflect on the fact that students are not tabulae rasae on the first day of class. Differences in experiences and values might create such different starting points for most students, compared with the minority whose backgrounds have adapted them to be ready for our unique demands, that "rising to the challenge" and "wrestling with the problem" are meaningless aphorisms and useless advice when they ask for help. Consequently, one of the fundamental components of pedagogical content knowledge has been to understand student understanding [8-10].

Attention to pedagogical content knowledge can be used in nearly all aspects of the educational process. Magnusson, Krajcik and Borko [8], in particular, have explicitly subdivided PCK into its meaningful (and useful) components. We have described in detail some of the philosophical and instructional strategies that have played a role in our own chemistry program, many of which draw from an applied PCK framework [11-15]. A few representative examples are outlined below.

\section{The PCK component in instructional settings}

\section{(1) Classroom time: Emphasizing the role of representation in chemistry [14-17].}

All disciplines attribute specialized meaning to words and other symbols. Although it is possible to demonstrate this idea by writing " $\mathrm{H}_{2} \mathrm{SO}_{4}$ " on the blackboard and making the point that it is not sulfuric acid, but only its representation (including the implicit lessons about the use of the " $\mathrm{H}$ ", the " $\mathrm{S}$ ", the "O" and the numerals to represent atoms and their relative ratios), it is not a good example for beginners. The symbol is too exclusively associated with the specialized context of chemistry, so there are no contexts within a student's experience (or anybody else's) that allows another attribution to " $\mathrm{H}_{2} \mathrm{SO}_{4}$ ". We 
begin our course by writing "HI" or "NO" on the blackboard and lead the class in a discussion of how important it is to attach assumptions to interpretations. For our part, we have needed to shift our initial questions from "What does 'HI' mean?" to "What could 'HI' represent?" We ask our students to shift from answers such as "'HI' is a greeting (or part of another word, or a portion of the alphabet, or hydroiodic acid)" to "If these Arabic letters are an English language word, then ' $\mathrm{H}-\mathrm{I}$ ' represents an informal form of the greeting 'hello'." After a while, our classroom language begins to shift to phrases that begin "What chemists mean by..." as even more sophisticated representational systems are encountered, such as depicting three-dimensional structures on two-dimensional surfaces (regardless of whether those surfaces are chalkboards, overhead transparencies, or pixelized displays). At first glance, the "HI" example may seem self-evident, perhaps even mundane, compared with using a sophisticated and pseudo-three-dimensional computational representation of an electrostatic surface to make the same point. However, the objective here is to introduce the concept of representations convincingly, where there are clearly alternative attributions that can be generated by students, before moving onto the kind of information-filled and complex imagery whose interpretation is as seemingly self-evident as "HI", but is only so to our experienced eyes.

\section{(2) Out-of-class time: Tacit understanding [11].}

Balancing options within the substitution-elimination manifold of reactions, as discussed previously, represents an opportunity to learn about the complexities of competitive mechanistic pathways. There is a fundamental mechanistic unity within the choices, which differ only in site of reactivity and the timing of the bonding changes. Except for cases where a pathway is structurally precluded (no B-elimination is possible for iodomethane), we have preceded the more subtle distinctions of substitution versus elimination and bimolecular versus unimolecular reactions with exercises that ask students to draw all of the possible substitution and elimination products from a given set of reagents [18]. The implicit, or tacit understanding of the changes involved in substitution and elimination reactions is a critical expectation in subsequent discussions about the possible competitions that occur. In an organic chemistry text that attempted a comprehensively different and mechanistic organization, Pine represented the unified view of substitution and elimination connectivity changes as an organizational principle (Form A in Figure 1) [19]. We have created and used extended versions of Form A to 


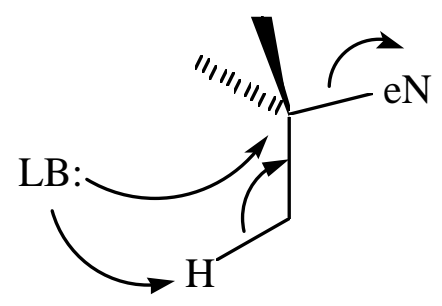

Form A

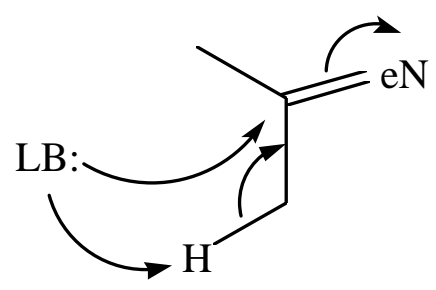

Form C

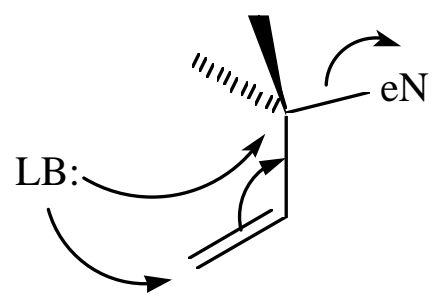

Form B

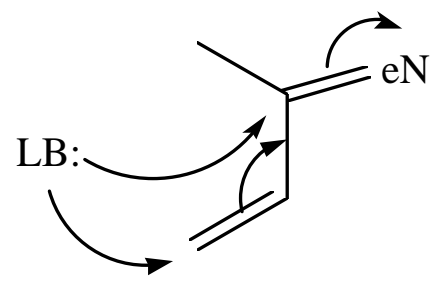

Form D

LB: = Lewis base

$\mathrm{eN}=$ an appropriate electronegative group

FIGURE 1. MECHANISTIC UNITY OF NUCLEOPHILIC SUBSTITUTION, ALLYLIC SUBSTITUTION, NUCLEOPHILIC 1,2- AND 1,4-ADDITION, B-ELIMINATION AND ENOLATE FORMATION.

discuss the even broader mechanistic unity of how Lewis bases interact with electrophiles in general.

\section{(3) Lab time: Cooperative and collaborative group work [20].}

We have taken the core question of relative identification, "Who has the Same Thing as I Do?," and created a blueprint for an activity in chemistry that has also been used by our colleagues in other disciplines [21]. Rather than following a prescribed set of directions towards one of a finite set of preordained results, our students collect and organize empirical data in order to answer the question: "Who has...?" Each member of the class selects one from a number of triplicated chemical samples, all examples of which have the same physical appearance (a group of powered solids; a set of clear, colorless liquids; aqueous acidic solutions that differ in concentration, and so on). The students are presented with a collection of experimental techniques that chemists use to obtain useful information from such samples. At this point, under the guided leadership of their instructor, the students formulate a group plan for the kind of data they will obtain, how they will obtain it, and how the information will be shared. The goal for 
these first-year students in their second week of college is quite specifically not to identify the molecular structure of their substance, because the strategies for doing this as far beyond their experience and ability to figure out. Instead, they answer the more immediately comprehensible question: based on the empirical data, who has the same substance as you do (and who does not)? This is an example of demonstrating an educational goal (how categorization and identification is done) within the context of chemistry that can be extended to other subjects. Identification tasks are de rigueur in organic chemistry laboratory courses, so how does this "Who has...?" activity derive from a PCK perspective? On the content side, the laboratory skills range from data collection using authentic strategies to devising methods for exchange and analysis; on the pedagogical side, the task has been designed so that both collaboration and cooperation are necessary in order to solve the problem, and we ensure that the activity is structured so that all participants remain honest to the objectives ( a simple example of which is keeping the stockroom coding scheme out of the hands of the faculty and lab instructors). Like the earlier example of using the Friedel-Crafts reaction [22], we have taken a traditional lab exercise and redesigned it in a way that retains an attention to the development of manipulative skills while also promoting higher order ("expert") skills [11].

\section{(4) Examinations: Literature-based problems.}

One of the goals we have set for our students is that they should be able to make sense of new and unfamiliar chemical examples in the context of their broader understanding. As described in detail elsewhere [15], one of the ways we demonstrate to students that memorization and recognition strategies alone are inadequate to accomplish this is to base all examination questions on examples drawn from the recent literature in a kind of "case study" approach. Starting with the first examination, students see that most problems have a citation to a recent journal article from the current year. This transmits the expectation better than anything else we could do that something beyond an intimate familiarity with the textbook examples is required. From the pedagogical perspective it also means that strict attention needs to be paid to in-class and out-of-class activities (including the strategies listed above) that explicitly model how one interacts with unfamiliar examples, which is the skill chemists use every time they open the latest issue of a journal, as they make sense of examples they have not seen before. Another PCK component is understanding how to format examination problems that are drawn from the literature (or from anywhere!) in a way that represents a fair assessment of the expectations of the course. In the same first-year Honors course alluded to earlier, a final 
examination we have developed takes the use of the literature to an even higher level: at the exam, the class receives photocopies of 4-5 short communications from a journal such as Tetrahedron Letters and an "examination paper" comprised of a series of questions based on information in the publications. The class is not provided with either the topics or the papers ahead of time. In fact, they are unaware of the format. Many other disciplines use the original works of authors with their undergraduate students, and devising ways to do this in beginning chemistry courses requires an understanding of student understanding in order to be fair as well as effective.

\section{Conclusion}

Subject matter mastery is the beginning, not the end, of effective instruction. If we are to design ways to help students (other than the predisposed ones) to understand chemistry and its place in the overall culture of intellectual inquiry, then pedagogical content knowledge is a useful tool for this. Recognizing the role of PCK allows us to focus past our own expertise and to identify the new things we can actively learn from an engaged participation in our own classrooms. What we learn from our students about how they learn can inform the way we design the instructional setting.

\section{ACKNOWLEDGEMENT}

The author thanks Joseph S. Krajcik (University of Michigan), James H. Wandersee (Louisiana State University) and Seyhan N. Ege (University of Michigan) for their helpful discussions and useful recommendations during the preparation of this manuscript.

\section{REFERENCES}

1. Herschbach, D. R. "Paradigms in Research and Parables in Teaching" J. Chem. Educ. 1993, 70, 391.

2. Agassi, J., in an essay reviewing "Science Teaching: The Role of History and Philosophy of Science" by Michael R. Matthews. Science \& Education 1996, 5, 69.

3. Ennis, R. H. "Critical Thinking and Subject Specificity: Clarification and Needed Research" Educational Researcher 1989, $18(\mathbf{8}), 4$.

4. McPeck, J. E. "Critical Thinking and Subject Specificity: A Reply to Ennis" Educational Researcher 1990, 19(4), 10. 
5. Ennis, R. E. "The Extent to Which Critical Thinking is Subject-Specific: Further Clarification" Educational Researcher 1990, 19(4), 13.

6. Shulman, L. S. "Those Who Understand: Knowledge Growth in Teaching" Educational Researcher 1986, 15, 4.

7. Shulman, L. S. "Knowledge and Teaching: Foundations of the New Reform" Harvard Educational Review 1987, 57, 1.

8. Magnusson, S.; Krajcik, J. S.; Borko, H. "Nature, Sources, and Development of Pedagogical Content Knowledge for Science Teaching." AETS Yearbook for 1996, in press.

9. Stevens, A. L.; Collins, A. "Multiple Conceptual Models of a Complex System" In, R. E. Sow, R. E.; Federico, P. A.; and Montague, W. E., Eds.; Aptitude, Learning and Instruction. Vol. 2: Cognitive Process Analyses of Learning and Problem Solving. Hillsdale, N.J.: Lawrence Erlbaum, 1980.

10. Champagne, A. B.; Klopfer, L. E.; Gunstone, R. F. "Cognitive Research and the Design of Science Instruction” Educational Psychologist 1982, 17, 31.

11. Coppola, B. P. ; Ege, S. N. ; Lawton, R. G. "The New Undergraduate Chemistry Curriculum at the University of Michigan. 2. Instructional Strategies and Assessment", J. Chem. Educ., submitted.

12. Ege, S. N.; Coppola, B. P. ; Lawton, R. G. "The New Undergraduate Chemistry Curriculum at the University of Michigan. 1. Philosophy, Curriculum, and the Nature of Change", J. Chem. Educ., in press.

13. Coppola, B. P.; Daniels, D. S. "Mea Culpa: Formal Education and the Dis-Integrated World" Science and Education, submitted.

14. Hoffmann, R.; Coppola, B. P. "Some Heretical Thoughts on What Our Students are Telling Us, Journal of College Science Teaching 1996, 25, 390.

15. Coppola, B. P.; Daniels, D. S. "Structuring the Liberal (Arts) Education in Chemistry", Chem. Educator 1996, 1 (2): S 1430-4171(96)02018-3. Avail. URL: http://journals.springerny.com/chedr.

16. Hoffmann, R. "Under the Surface of the Chemical Article," Angewandte Chemie 1988, 100, 16531663; Angewandte Chemie, (International Edition in English) 1988, 27, 1593.

17. Hoffmann, R. The Same and Not the Same New York: Columbia University Press, 1995.

18. Ege, S. N.; Kleinman, R.; Carter, M. L. C. "Workbook Problems" In, Study Guide for Organic Chemistry, 3rd Ed., Boston: D. C. Heath \& Co., 1994.

19. Pine, S. Organic Chemistry, 5th ed.; New York: McGraw Hill, 1987, p. 358. 
20. Coppola, B. P. "Progress in Practice: Exploring the Cooperative and Collaborative Dimensions of Group Learning." Chem. Educator 1996, 1 (1): S 1430-4171(96)01006-0. Avail. URL: http://journals.springer-ny.com/chedr.

21. Coppola, B. P.; Lawton, R. G. “'Who Has the Same Substance that I Have?' A Blueprint for Collaborative Learning Activities" J. Chem. Educ. 1995, 72, 1120.

22. Todd, D.; Pickering, M. “Three Puzzles for Organic Laboratory” J. Chem. Educ. 1988, 65, 1100. 